\title{
ANALYTIC FUNCTIONS WITH QUASI-ANALYTIC BOUNDARY VALUES $\left({ }^{1}\right)$
}

\author{
BY \\ WILLIAM A. GROENING
}

1. Introduction. Given a sequence of positive numbers $A_{1}, A_{2}, \ldots$, let $F\left(A_{n}\right)$ be the set of functions $f=\sum a_{n} z^{n}$ analytic in the disc $D=\{z:|z|<1\}$ such that

(i) $f^{(k)}$ has a continuous extension to $D^{-}$for $k=0,1, \ldots$,

(ii) $\left\{a_{n} A_{n}\right\}$ is a bounded sequence.

We call $F\left(A_{n}\right)$ quasi-analytic if $f \in F\left(A_{n}\right)$ and $f^{(k)}(1)=0(k=0,1, \ldots)$ together imply that $f \equiv 0$. The main purpose of this paper is to give a necessary and sufficient condition on the sequence $\left\{A_{n}\right\}$ for $F\left(A_{n}\right)$ to be quasi-analytic (Theorem 3.4) and to extend the results to functions analytic in the half-plane and functions of several variables.

In $\$ 2$ we prove a quasi-analytic theorem on a related class of functions. This theorem is stated without proof by B. I. Korenbljum in [3] and [4]. $\$ 3$ is devoted to the main Theorem 3.4: If $\log A_{n}$ is convex in $\log n$, then $F\left(A_{n}\right)$ is quasi-analytic if and only if $\sum_{1}^{\infty} n^{-3 / 2} \log A_{n}$ diverges. Half of Theorem 3.4 is proven by a slightly different method in [2, p. 331]. There Carleson shows thät if $\log A_{n}$ is convex in $\log n$ and $\sum_{1}^{\infty} n^{-3 / 2} \log A_{n}$ diverges then functions in $F\left(A_{n}\right)$ can have only finitely many zeros. The first step of his proof reduces this problem to showing that $F\left(A_{n}\right)$ must be quasi-analytic.

In $\$ 4$ we give a half-plane analogue of Theorem 3.4 by looking at functions of the form $f(z)=\int_{0}^{\infty} \hat{f}(t) e^{i t z} d t$, where $\hat{f}(t)$ is a measurable function and $\hat{f}(t) A(t)$ is bounded. In $\S 5$ we give one possible analogue of Theorem 3.4 to functions of several variables. This result is obtained by applying the one variable theorem.

2. Functions with bounded derivatives. Since we make use of the following computation throughout this paper, we state it here in the form of a lemma.

Lemma 2.1. Suppose that $f$ is analytic in the unit disc $D, f^{(k)}$ has a continuous extension to $D^{-}$and $f^{(k)}(1)=0$ for $k=0,1, \ldots, n-1$. Then for $k=0,1, \ldots, n-1$ and $z_{0}$ in $D^{-}$,

$$
f^{(k)}\left(z_{0}\right)=\frac{1}{(n-k-1) !} \int_{1}^{z_{0}}\left(z_{0}-z\right)^{n-k-1} f^{(n)}(z) d z .
$$

Proof. Write $f^{(k)}\left(z_{0}^{\prime}\right)=\int_{1}^{z_{0}} f^{(k+1)}(z) d z$ and integrate by parts.

Presented to the Society, January 23, 1969; received by the editors October 1, 1968 and, in revised form, December 5, 1968.

(1) This paper is essentially a portion of a doctoral dissertation written under the guidance of Professor Peter L. Duren at the University of Michigan while the author was a NSF Trainee. 
Given a sequence $\left\{M_{n}\right\}$ of positive numbers, let $C\left(M_{n}\right)$ be the set functions $f$ analytic in the disc $D=\{z:|z|<1\}$ such that for $n=0,1, \ldots$,

(i) $f^{(n)}$ has a continuous extension to $D^{-}$;

(ii) $\left|f^{(n)}(z)\right| \leqq B M_{n}$ in $D$ for some number $B=B(f)$.

The class $C\left(M_{n}\right)$ is said to be quasi-analytic if $f \in C\left(M_{n}\right)$ and $f^{(n)}(1)=0(n=0,1$, ...) together imply that $f \equiv 0$.

TheOREM 2.2 (KoREnBluUM). Let $T(r)=\sup _{n \geqq 0} r^{n} M_{n}^{-1}$. The class $C\left(M_{n}\right)$ is quasi-analytic if and only if $\int_{1}^{\infty} r^{-3 / 2} \log T(r) d r$ diverges.

Proof. Suppose that the integral converges. Then by Theorem 3 of [3] there is a function $F(w) \not \equiv 0$ analytic in the right half-plane $H$ such that $F^{(n)}$ has a continuous extension to $H^{-},\left|F^{(n)}(w)\right| \leqq M_{n}$ in $H$ and $F^{(n)}(0)=0$ for $n=0,1, \ldots$ Let $f(z)=$ $F(1-z)$. Then $f$ is in $C\left(M_{n}\right)$ and $f^{(n)}(1)=0$ for $n=0,1, \ldots$; but $f \not \equiv 0$. Hence $C\left(M_{n}\right)$ is not quasi-analytic.

Conversely, suppose that the integral diverges and $f$ is a function in $C\left(M_{n}\right)$ such that $f^{(n)}(1)=0, n=0,1, \ldots$ Computing the derivatives of $F(w)=f((1-w) /(1+w))$ gives an expression of the form

$$
F^{(n)}(w)=\sum_{k=1}^{n} a_{n k}(1+w)^{-(n+k)} f^{(k)}\left(\frac{1-w}{1+w}\right)
$$

where the $a_{n k}$ are real numbers. Differentiating this expression and comparing coefficients, we obtain the formula

$$
\begin{aligned}
a_{n+1 k} & =-(n+k) a_{n k}-2 a_{n k-1}, \\
a_{n 0} & =a_{n n+1}=0, \quad\left|a_{n n}\right|=2^{n} .
\end{aligned}
$$

Using the binomial expansion

$$
(n+1)^{2(n+1-k)}=n^{2(n+1-k)}+2(n+1-k) n^{2(n+1-k)-1}+\cdots,
$$

an induction argument on $n$ shows that $\left|a_{n k}\right| \leqq 2^{n} n^{2(n-k)} /(n-k)$ !. For $k=0, \ldots, n-1$ we have

$$
f^{(k)}\left(z_{0}\right)=\frac{1}{(n-k-1) !} \int_{1}^{z_{0}}\left(z_{0}-z\right)^{n-k-1} f^{(n)}(z) d z
$$

by Lemma 2.1. Hence

$$
\left|f^{(k)}\left(z_{0}\right)\right| \leqq \frac{1}{(n-k-1) !} 2^{n-k} B M_{n} .
$$

And

$$
\begin{aligned}
\left|F^{(n)}(w)\right| & \leqq \sum_{k=1}^{n} \frac{a_{n k}}{|1+w|^{n+k}}\left|f^{(k)}\left(\frac{1-w}{1+w}\right)\right| \\
& \leqq \sum_{k=1}^{n-1} 2^{n} \frac{n^{2(n-k)}}{(n-k) !} \frac{2^{n-k}}{(n-k-1) !} B M_{n}+2^{n} B M_{n} .
\end{aligned}
$$


Now the first term is bounded by

$$
4^{n} B M_{n} \sum_{k=1}^{n-1} \frac{n^{2(n-k)}}{[(n-k-1) !]^{2}}=4^{n} B M_{n} \sum_{k=0}^{n-2} \frac{n^{2 k+2}}{(k !)^{2}} .
$$

So we conclude that

$$
\left|F^{(n)}(w)\right| \leqq 4^{n} B M_{n} n^{2} \sum_{k=0}^{n}\left(\frac{n^{k}}{k !}\right)^{2} \leqq 4^{n} B M_{n} n^{2}\left(e^{n}\right)^{2} .
$$

Again referring to Theorem 3 of [3] we conclude that $F \equiv 0$ and hence $f \equiv 0$.

We define a class of functions similar to $C\left(M_{n}\right)$ as follows. If $f$ is analytic in $D$ and has a continuous extension to $D^{-}$, denote the boundary values by $\tilde{f}(\theta)=f\left(e^{i \theta}\right)$. Let $\widetilde{C}\left(M_{n}\right)$ be the set of functions analytic on $D$ such that for each $n=0,1, \ldots$,

(i) $f^{(n)}$ has a continuous extension to $D^{-}$;

(ii) $\left|\tilde{f}^{(n)}(\theta)\right| \leqq B M_{n}$ on $[0,2 \pi]$.

The class $\widetilde{C}\left(M_{n}\right)$ is said to be quasi-analytic if $f \in \widetilde{C}\left(M_{n}\right)$ and $\tilde{f}^{(n)}(0)=0(n=0,1, \ldots)$ together imply that $f \equiv 0$. Estimates similar to those in the proof of Theorem 2.2 show that $\widetilde{C}\left(M_{n}\right)$ is quasi-analytic if and only if $C\left(M_{n}\right)$ is.

3. Functions with small Taylor coefficients. Mandelbrojt [6] has shown that if $g(\theta)=\sum_{-\infty}^{\infty} a_{n} e^{i n \theta}$ is infinitely differentiable, $\left\{a_{n} A_{|n|}\right\}_{-\infty}^{\infty}$ is bounded, and $g^{(n)}(0)=0$ $(n=0,1, \ldots)$, then a necessary and sufficient condition to conclude that $g \equiv 0$ is that $\sum_{n=1}^{\infty} n^{-2} \log A_{n}=\infty$. The main theorem of this section, Theorem 3.4, says that if $g(\theta)$ comes from the boundary values of an analytic function, then the appropriate condition is that $\sum_{n=1}^{\infty} n^{-3 / 2} \log A_{n}=\infty$. Thus by making an additional assumption on the functions, we can relax the condition on the sequence $\left\{A_{n}\right\}$. The methods we use are much the same as the methods Mandelbrojt uses.

In this section, $A_{1}, A_{2}, \ldots$ will be a sequence of positive numbers such that $\log A_{n}$ is convex in $\log n$, i.e.

$$
s_{n}=\frac{\log A_{n+1}-\log A_{n}}{\log (n+1)-\log n}
$$

is a nondecreasing sequence. We let $F\left(A_{n}\right)$ be the set of functions $f(z)=\sum_{k=0}^{\infty} a_{k} z^{k}$ analytic in $D$ such that $f^{(n)}(z)$ has a continuous extension to $D^{-}(n=0,1, \ldots)$ and such that $\left\{A_{k} a_{k}\right\}$ is bounded. We call $F\left(A_{n}\right)$ quasi-analytic if $f \in F\left(A_{n}\right)$ and $f^{(n)}(1)=0(n=0,1, \ldots)$ together imply that $f \equiv 0$.

We begin by proving some properties of the sequence $\left\{A_{n}\right\}$.

LEMMA 3.1. If $\lim _{n \rightarrow \infty} s_{n}=\infty$, then for each nonnegative integer $k,\left\{n^{k} A_{n}^{-1}\right\}$ is a bounded sequence.

Proof. Fix $k$ and choose $n_{0}$ so that $s_{n_{0}} \geqq k$. Then for $n \geqq n_{0}$,

$$
\begin{aligned}
\log A_{n+1}-\log A_{n} & =s_{n}(\log (n+1)-\log n) \\
& \geqq k(\log (n+1)-\log n) .
\end{aligned}
$$

Hence $n^{k} A_{n}^{-1} \geqq(n+1)^{k} A_{n+1}^{-1}$; and $n^{k} A_{n}^{-1}$ is nonincreasing for $n \geqq n_{0}$. 
This lemma allows us to state the following proposition which is crucial in relating $F\left(A_{n}\right)$ to a class of functions of the type $\widetilde{C}\left(M_{n}\right)$.

Proposition 3.2. Suppose that $s_{n} \geqq 0$ and $\lim _{n \rightarrow \infty} s_{n}=\infty$. Let $c(n)=\sup _{k \geqq 1} k^{n} A_{k}^{-1}$ and let $d(t)=\sup _{n \geqq 1} t^{n} c(n)^{-1}$. Then for $n=1,2, \ldots, d(n) \leqq A_{n} \leqq n d(n)$. Furthermore, given $n_{0}$, there exists a number $t_{n_{0}}$ such that for $t \geqq t_{n_{0}}, d(t)=\sup _{n \geqq n_{0}} t^{n} c(n)^{-1}$.

Proof. By the previous lemma $c(n)$ is finite, so that the definition of $d(t)$ is meaningful. For fixed $n$ and for each $k, A_{n} \geqq n^{k} c(k)^{-1}$. Hence $A_{n} \geqq d(n)$.

To prove the second inequality, fix $n$ and let $k$ be the integer such that $k \leqq s_{n}$ $<k+1$. An argument similar to the proof of Lemma 3.1 shows that for $j \geqq n$,

$$
j^{k} A_{j}^{-1} \leqq n^{k} A_{n}^{-1}<n^{k+1} A_{n}^{-1} \text {. }
$$

While, for $1 \leqq j<n$,

$$
\begin{aligned}
\log A_{j+1}-\log A_{j} & =s_{j}(\log (j+1)-\log j) \\
& <(k+1)(\log (j+1)-\log j) .
\end{aligned}
$$

So that

$$
j^{k+1} A_{j}^{-1}<(j+1)^{k+1} A_{j+1}^{-1} \leqq n^{k+1} A_{n}^{-1} .
$$

We therefore have $c(k)=\sup _{j \geqq 1} j^{k} A_{j}^{-1} \leqq n^{k+1} A_{n}^{-1}$. So that $A_{n} \leqq n^{k+1} c(k)^{-1} \leqq n d(n)$.

Finally, given $n_{0}$ choose $t_{n_{0}} \geqq 1$ so that $t_{n_{0}} c(n)>c\left(n_{0}\right)$ for each $n$ less than $n_{0}$. Now fix $t \geqq t_{n_{0}}$. For $n<n_{0}$,

$$
t^{n_{0}} c(n) \geqq t^{n+1} c(n) \geqq t^{n} t_{n_{0}} c(n)>t^{n} c\left(n_{0}\right) .
$$

Thus $t^{n_{0}} c\left(n_{0}\right)^{-1}>t^{n} c(n)^{-1}$. And hence for $t \geqq t_{n_{0}}$,

$$
d(t)=\sup _{n \geqq 0} t^{n} c(n)^{-1}=\sup _{n \geqq n_{0}} t^{n} c(n)^{-1} .
$$

Before proving the main theorem of this section, we prove the following special case which contains the crux of the argument.

THEOREM 3.3. Suppose that $s_{n} \geqq 0$ and $\lim _{n \rightarrow \infty} s_{n}=\infty$. Then a necessary and sufficient condition for $F\left(A_{n}\right)$ to be quasi-analytic is that

$$
\sum_{n=1}^{\infty} n^{-3 / 2} \log A_{n}
$$

diverge.

Proof. Sufficiency. We first show that $F\left(A_{n}\right)$ is contained in $\widetilde{C}\left(M_{n}\right)$ where $M_{n}=c(n+2)$. Suppose $f$ is in $F\left(A_{n}\right)$. Then $f(z)=\sum_{n=0}^{\infty} a_{n} z^{n}$ where $\left|a_{n}\right| \leqq B A_{n}^{-1}$ for some constant $B$. Now $f^{f}(\theta)=\sum_{n=0}^{\infty} a_{n} e^{i n \theta}$. So for $k=1,2, \ldots$,

$$
\begin{aligned}
\left|f^{(k)}(\theta)\right| & =\left|\sum_{n=0}^{\infty} a_{k}(i n)^{k} e^{i n \theta}\right| \leqq \sum_{n=1}^{\infty}\left|a_{n}\right| n^{k} \\
& \leqq \sum_{n=1}^{\infty} n^{-2} n^{k+2} B A_{n}^{-1} \leqq B c(k+2) \sum_{n=1}^{\infty} n^{-2} .
\end{aligned}
$$

Hence $f \in \widetilde{C}\left(M_{n}\right)$ where $M_{n}=c(n+2)$. 
Therefore it is enough to show that the divergence of (1) implies that $\widetilde{C}\left(M_{n}\right)$ is quasi-analytic, i.e. that $\int_{1}^{\infty} r^{-3 / 2} \log T(r)$ diverges. Now

$$
T(r)=\sup _{k \geqq 0} r^{k} c(k+2)^{-1}=r^{-2} \sup _{k \geqq 0} r^{k+2} c(k+2) .
$$

So for $r \geqq t_{2}, T(r)=r^{-2} d(r)$. By Proposition 3.2 the divergence of (1) is equivalent to the divergence of $\sum_{1}^{\infty} n^{-3 / 2} \log d(n)$ and hence to the divergence of

$$
\int_{1}^{\infty} r^{-3 / 2} \log T(r)
$$

Necessity. Let $M_{0}=1$ and $M_{k}=c(k-1)$ for $k=1,2, \ldots$ We first show that the convergence of (1) implies that $\tilde{C}\left(M_{n}\right)$ is not quasi-analytic. Let $T(r)=\sup _{k \geqq 0} r^{k} M_{k}^{-1}$. Then for $r \geqq M_{1}$,

$$
T(r)=\sup _{k \geqq 0} r^{k} M_{k}^{-1}=r \sup _{k \geqq 1} r^{k-1} c(k-1)^{-1}=r d(r) .
$$

By an argument similar to the above, the convergence of the series (1) implies that $\int_{1}^{\infty} r^{-3 / 2} \log T(r)$ converges and therefore $\widetilde{C}\left(M_{n}\right)$ is not quasi-analytic.

Hence there is a function $f$ in $\widetilde{C}\left(M_{n}\right)$ such that $\tilde{f}^{(n)}(0)=0(n=0,1, \ldots)$ but $f \not \equiv 0$. The proof of the theorem will be complete if we show that $f$ is also in $F\left(A_{n}\right)$. Fix $n \geqq 1$. Integrating by parts we find that

And

$$
a_{n}=\frac{1}{2 \pi} \int_{0}^{2 \pi} \tilde{f}(\theta) e^{-i n \theta} d \theta=\cdots=\frac{1}{2 \pi} \int_{0}^{2 \pi} \tilde{f}^{(k)}(\theta)\left(\frac{1}{i n}\right)^{k} e^{-i n \theta} d \theta .
$$

$$
\left|a_{n}\right| \leqq B M_{k}\left(\frac{1}{n}\right)^{k} \leqq \frac{B}{n} \frac{1}{d(n)} \leqq \frac{B}{A_{n}} .
$$

Therefore $\left\{\left|a_{n}\right| A_{n}\right\}$ is bounded and $f$ is in $F\left(A_{n}\right)$.

Theorem 3.3 can be improved to give the main result of this section.

THEOREM 3.4. If $\log A_{n}$ is a convex sequence in $\log n$, then a necessary and sufficient condition for $F\left(A_{n}\right)$ to be quasi-analytic is that

$$
\sum_{n=1}^{\infty} n^{-3 / 2} \log A_{n}
$$

diverge.

Proof. First suppose that $\lim _{n \rightarrow \infty} s_{n}=\infty$. If $s_{n} \geqq 0(n=1,2, \ldots)$, then Theorem 3.3 applies. If not, then since $\lim _{n \rightarrow \infty} s_{n}=\infty$, there is an integer $n_{0}$ such that $s_{n} \geqq 0$ for all $n \geqq n_{0}$. Let

Then

$$
\begin{aligned}
& \bar{A}_{n}=A_{n_{0}} \text { if } n \leqq n_{0} \text {, } \\
& =A_{n} \quad \text { if } n \geqq n_{0} \text {. }
\end{aligned}
$$

$$
\begin{aligned}
\bar{s}_{n}=0 & \text { if } n<n_{0}, \\
=s_{n} & \text { if } n \geqq n_{0},
\end{aligned}
$$


where

$$
\bar{s}_{n}=\frac{\log \bar{A}_{n+1}-\log \bar{A}_{n}}{\log (n+1)-\log n}
$$

Hence $\log \bar{A}_{n}$ is convex in $\log n, \bar{s}_{n} \geqq 0$ and $\lim _{n \rightarrow \infty} \bar{s}_{n}=\infty$. So Theorem 3.3 applies to $F\left(A_{n}\right)$. But $F\left(\bar{A}_{n}\right)=F\left(A_{n}\right)$ and $\sum_{1}^{\infty} n^{-3 / 2} \log \bar{A}_{n}$ diverges if and only if (1) diverges. Hence the theorem is true whenever $\lim _{n \rightarrow \infty} s_{n}=\infty$.

Now suppose that it is not the case that $\lim _{n \rightarrow \infty} s_{n}=\infty$. Then, since $\left\{s_{n}\right\}$ is a nondecreasing sequence, we must have $s_{n} \leqq b$ for some number $b$. In this case we show (1) converges and $F\left(A_{n}\right)$ is not quasi-analytic. Since

$$
\begin{aligned}
\log A_{n+1}-\log A_{n} & =s_{n}(\log (n+1)-\log n) \\
& \leqq b(\log (n+1)-\log n),
\end{aligned}
$$

we have

$$
A_{n+1} \leqq A_{n}\left(\frac{n+1}{n}\right)^{b} \leqq A_{n-1}\left(\frac{n}{n-1}\right)^{b}\left(\frac{n+1}{n}\right)^{b} \leqq \cdots \leqq A_{1}(n+1)^{b} .
$$

And

$$
\sum_{1}^{\infty} n^{-3 / 2} \log A_{n} \leqq \sum_{1} n^{-3 / 2}\left(\log A_{1}+b \log n\right)<\infty .
$$

To show $F\left(A_{n}\right)$ is not quasi-analytic we exhibit a non-quasi-analytic class $F\left(\bar{A}_{n}\right)$ which is contained in $F\left(A_{n}\right)$. Let $\bar{A}_{n}=A_{1} n^{b \log n}$. Since $A_{n} \leqq A_{1} n^{b} \leqq \bar{A}_{n}$ for $n \geqq 3$, $F\left(\bar{A}_{n}\right)$ is contained in $F\left(A_{n}\right)$. And since

$$
\bar{s}_{n}=\frac{\log \bar{A}_{n+1}-\log \bar{A}_{n}}{\log (n+1)-\log n}=b(\log (n+1)+\log n),
$$

we may apply Theorem 3.3 to $F\left(\bar{A}_{n}\right)$. But

$$
\sum_{1}^{\infty} n^{-3 / 2} \log \bar{A}_{n}=\sum_{1}^{\infty} n^{-3 / 2}\left(\log A_{1}+b(\log n)^{2}\right)<\infty
$$

So $F\left(\bar{A}_{n}\right)$ is not quasi-analytic.

A number of comments on Theorem 3.4 should be made. The first is that if the series (1) diverges, then any function $f(z)=\sum_{0}^{\infty} a_{k} z^{k}$, such that $\left\{a_{k} A_{k}\right\}$ is bounded, is already in $F\left(A_{n}\right)$, i.e. $f^{(n)}(z)$ has a continuous extension to $D^{-}$for $n=0,1, \ldots$ For as we have seen, the divergence of (1) implies that $\lim _{n \rightarrow \infty} s_{n}=\infty$. Thus, by Lemma 3.1, $\left\{n^{k+2} A_{n}^{-1}\right\}$ is bounded for each $k$. Hence $f^{(k)}(z)=\sum_{n=k}^{\infty} a_{n} z^{n-k} n ! /(n-k) !$ converges uniformly on $D^{-}$, since

$$
\left|\frac{n !}{(n-k) !} a_{n}\right| \leqq n^{k}\left|a_{n}\right| \leqq n^{-2} n^{k+2} B A_{n}^{-1} \leqq n^{-2} B_{1} .
$$

Secondly, one might want to study the classes $F^{p}\left(A_{n}\right)(0<p<\infty)$, where $F^{p}\left(A_{n}\right)$ is the set of functions $f(z)=\sum_{0}^{\infty} a_{k} z^{k}$ analytic in $D$ such that $f^{(n)}$ has a continuous 
extension to $D^{-}(n=0,1, \ldots)$ and $\sum_{1}^{\infty}\left|a_{k}\right|^{p} A_{k}<\infty$. The following theorem shows, however, that the problem reduces to considering $F\left(A_{n}\right)$.

THEOREM 3.5. $F\left(n^{2 / p} A_{n}^{1 / p}\right) \subset F^{p}\left(A_{n}\right) \subset F\left(A_{n}^{1 / p}\right)$; and hence $F^{p}\left(A_{n}\right)$ is quasi-analytic if and only if (1) diverges.

The proof of this theorem is straightforward and is left to the reader.

4. An analogue in the half-plane. The class $F\left(A_{n}\right)$ has a "continuous analogue" in the following sense. Given a real-valued function $A(t)$ defined on the open interval $(0, \infty)$, let $F(A(t))$ be the set of all functions $f$ analytic in the upper halfplane $H=\{z: \operatorname{Im}(z)>0\}$ such that

(i) $f^{(n)}$ has a continuous extension to $H^{-}(n=0,1, \ldots)$,

(ii) $f$ is of the form $f(z)=\int_{0}^{\infty} e^{i t z} \hat{f}(t) d t$, where $\hat{f}(t)$ is a measurable function and $|\hat{f}(t)| A(t)$ is bounded.

We call $F(A(t))$ quasi-analytic if $f \in F(A(t))$ and $f^{(n)}(0)=0(n=0,1, \ldots)$ together imply $f \equiv 0$.

Thus, looking at the boundary values of functions in $F(A(t))$ and $F\left(A_{n}\right)$, functions in $F(A(t))$ have Fourier transforms which vanish on the negative real line and go rapidly to zero on the positive real line, while the negative Fourier coefficients of functions in $F\left(A_{n}\right)$ vanish and the positive Fourier coefficients go rapidly to zero.

We restrict our attention to functions $A(t)$ for which $\log A(t)$ is a convex function of $\log t, A(t)$ is bounded below by 1 , and $A(t)$ is bounded on the interval $(0,1]$. Such a function has the following properties:

(i) For $t_{0}<t_{1}<t_{2}$,

(ii) For $h>0$,

$$
\begin{aligned}
\frac{\log A\left(t_{1}\right)-\log A\left(t_{0}\right)}{\log t_{1}-\log t_{0}} & \leqq \frac{\log A\left(t_{2}\right)-\log A\left(t_{0}\right)}{\log t_{2}-\log t_{0}} \\
& \leqq \frac{\log A\left(t_{2}\right)-\log A\left(t_{1}\right)}{\log t_{2}-\log t_{1}}
\end{aligned}
$$

$$
f(t ; h)=\frac{\log A(t+h)-\log A(t)}{\log (t+h)-\log t}
$$

is a decreasing function of $h$.

(iii) $s(t)=\lim _{h \rightarrow 0^{+}} f(t ; h)$ exists and is a nondecreasing function of $t$.

(iv) For $t<t_{0}$,

$$
\frac{\log A\left(t_{0}\right)-\log A(t)}{\log t_{0}-\log t} \leqq s\left(t_{0}\right) .
$$

Properties (i) and (ii) are statements about slopes of chords of the graph of $\log A(t)$ vs. $\log t$ and are clear when viewed geometrically. The function $s(t)$ measures the slope of the graph at $(\log t, \log A(t))$. The corresponding properties for convex functions are known $[3, \S 3.18]$, so we omit a proof of the above properties. 
LEMMA 4.1. If $\lim _{t \rightarrow \infty} s(t)=\infty$, then for each nonnegative integer $k, t^{k} A(t)^{-1}$ is bounded.

Proposition 4.2. Suppose $s(t) \geqq 0$ and $\lim _{t \rightarrow \infty} s(t)=\infty$. Let $c(n)=\sup _{t \geqq 1} t^{n} A(t)^{-1}$ and $d(t)=\sup _{n \geqq 0} t^{n} c(n)^{-1}$. Then for $t \geqq 1, d(t) \leqq A(t) \leqq t d(t)$. Furthermore, given $n_{0}$ there exists a number $t_{n_{0}}$ such that for $t \geqq t_{n_{0}}, d(t)=\sup _{n \geqq n_{0}} t^{n} c(n)^{-1}$.

The proofs of the lemma and proposition follow the corresponding proofs given in the previous section. It is interesting to note that if we let $c(r)=\sup _{t \geqq 1} t^{r} A(t)^{-1}$ $(0 \leqq r<\infty)$ and $d(t)=\sup _{r \geqq 0} t^{r} c(r)^{-1}$, then $d(t)=A(t)$.

TheOREM 4.3. Suppose that $s(t) \geqq 0$ and $\lim _{t \rightarrow \infty} s(t)=\infty$. Then a necessary and sufficient condition for $F(A(t))$ to be quasi-analytic is that

$$
\int_{1}^{\infty} t^{-3 / 2} \log A(t) d t
$$

diverge.

Proof. Sufficiency. Suppose that $f$ is in $F(A(t))$ and $f^{(n)}(0)=0,1, \ldots$ Since $t^{n+2} A(t)^{-1} \leqq c(n+2)$ for $t \geqq 1$, the integral

$$
f^{(n)}(z)=\int_{0}^{\infty}(i t)^{n} e^{i t z} \hat{f}(t) d t
$$

converges uniformly for $\operatorname{Im}(z) \geqq 0$. In fact,

$$
\begin{aligned}
\left|f^{(n)}(z)\right| & \leqq \int_{0}^{\infty} t^{n} B A(t)^{-1} d t \\
& \leqq B+\int_{1}^{\infty} t^{-2} t^{n+2} B A(t)^{-1} d t \\
& \leqq B+B c(n+2) \int_{1}^{\infty} t^{-2} d t
\end{aligned}
$$

for $n=0,1, \ldots$ If we let

$$
T(r)=\sup _{n \geqq 0} r^{n} c(n+2)^{-1}=r^{-2} \sup _{n \geqq 2} r^{n+2} c(n+2)^{-1},
$$

then $T(r)=r^{-2} d(r) \geqq r^{-3} A(r)$ for $r \geqq t_{2}$. Hence the divergence of (2) implies that $\int_{1}^{\infty} r^{-3 / 2} \log T(r) d r$ diverges. Hence by Theorem 3 of [3], $f \equiv 0$.

Necessity. Let $M_{0}=1$ and $M_{n}=e^{-n} c(n-1), n=1,2, \ldots$ As in the proof of Theorem 3.3, the convergence of (2) implies that $\int_{1}^{\infty} r^{-3 / 2} \log T(r) d r$ diverges, where $T(r)=\sup _{n \geqq 0} r^{n} M_{n}^{-1}$. Hence by Theorem 3 of [3] there is a function $f \not \equiv 0$ analytic in the upper half-plane $H$ such that $f^{(n)}$ has a continuous extension to $H^{-}$, $\left|f^{(n)}\right| \leqq M_{n}$ and $f^{(n)}(0)=0$ for $n=0,1, \ldots$ Let $g(z)=(z+i)^{-2} f(z)$ then $g$ has the same properties as $f$. The proof will be complete if we show that $g$ is in $F(A(t))$.

$$
g^{(n)}(z)=\sum_{k=0}^{n} C_{n, k} f^{(n-k)}(z)(z+i)^{-(2+k)}(-1)^{k}(k+1) !
$$


By Lemma 2.1

$$
f^{(n-k)}(z)=\frac{1}{(k-1) !} \int_{0}^{z}(z-w)^{k-1} f^{(n)}(w) d w
$$

Hence

$$
\left|f^{(n-k)}(z)\right| \leqq \frac{1}{(k-1) !} z^{k} B M_{n}, \quad k=1,2, \ldots
$$

And

$$
\left|g^{(n)}(z)\right| \leqq C_{n, 0}\left|f^{(n)}(z)\right||z+i|^{-2}+\left[\sum_{k=1}^{n} C_{n, k} \frac{1}{(k-1) !}|z|^{k} B M_{n}\right]\left[|z+i|^{-(2+k)}(k+1) !\right]
$$

Since the second term is less than

$$
\sum_{k=1}^{n} C_{n, k} B M_{n}|z+i|^{-2}(k+1) k
$$

we have

$$
\left|g^{(n)}(z)\right| \leqq B_{1} e^{n} M_{n}|z+i|^{-2} .
$$

In particular, $g$ is in the class $H^{2}$ of the upper half-plane. Therefore by the PaleyWiener theorem, the Fourier transform $\hat{g}(t)$ vanishes for $t<0$. For $t>0$,

$$
2 \pi \hat{g}(t)=\int_{-\infty}^{\infty} e^{-i t x} g(x) d x=\int_{-\infty}^{\infty}(-i t)^{-n} g^{(n)}(x) e^{i t x} d x
$$

So that

$$
|2 \pi \hat{g}(t)| \leqq t^{-n} B e^{n} M_{n} \int_{-\infty}^{\infty}|x+i|^{-2} d x
$$

Hence for $t \geqq 1$,

$$
\begin{aligned}
\hat{g}(t) & \leqq B_{1} \inf _{n \geqq 1} t^{-n} e^{n} M_{n}=B_{1} t^{-1} \inf _{n \geqq 0} t^{-n} c(n) \\
& \leqq B_{1} t^{-1} d(t)^{-1} \leqq B_{2} A(t)^{-1}
\end{aligned}
$$

And for $0<t<1$,

$$
|\hat{g}(t)| \leqq B_{1} M_{0} \leqq B_{2} A(t)^{-1},
$$

since $A(t)$ is bounded on the interval $(0,1)$. Thus $g$ is in $F(A(t))$.

As before, we immediately improve Theorem 4.3 to obtain the main result.

THEOREM 4.4. Suppose that $A(t)$ is defined and bounded below by 1 on the interval $[0, \infty)$. Suppose further that $A(t)$ is bounded on $(0,1)$ and that $\log A(t)$ is a convex function of $\log t$. Then a necessary and sufficient condition for $F(A(t))$ to be quasianalytic is that

$$
\int_{1}^{\infty} t^{-3 / 2} \log A(t) d t
$$

diverge. 
Proof. If $\lim _{t \rightarrow \infty} s(t)=\infty$, the proof proceeds as in the previous section. If not, then let $a=\sup _{0 \leqq t \leqq e} A(t)$ and choose $b$ so that $b \geqq s(t)$ for all $t$. Let

$$
\begin{aligned}
\bar{A}(t) & =a t^{b \log t} & & \text { for } t \geqq e, \\
& =a e^{b} & & \text { for } t \leqq e .
\end{aligned}
$$

Then as in the previous section $F(\bar{A}(t))$ is contained in $F(A(t))$ and $F(\bar{A}(t))$ is not quasi-analytic. Hence $F(A(t))$ is not quasi-analytic. On the other hand (2) converges.

We again observe that if (2) diverges, then any function $f$ analytic in the upper half-plane $H$ and of the form $f(z)=\int_{0}^{\infty} \hat{f}(t) e^{i t z} d t$ where $\hat{f}(t) A(t)$ is bounded, is already in $F(A(t))$. For the divergence of (2) implies that $\lim _{t \rightarrow \infty} s(t)=\infty$ and hence (3) shows that

$$
f^{(n)}(z)=\int_{0}^{\infty} \hat{f}(t)(i t)^{n} e^{i t z} d t
$$

converges uniformly on $H^{-}$. Hence $f^{(n)}(z)$ has a continuous extension to $H^{-}$.

5. Functions of several variables. Since we will be working in the space of $\boldsymbol{n}$ complex variables $C^{n}$, we wish to make use of multi-indices of nonnegative integers $m=\left(m_{1}, \ldots, m_{n}\right)$. If $m$ and $l$ are multi-indices, $r$ is an $n$-tuple of real or complex numbers, and $a$ and $b$ are real numbers, then

$$
\begin{aligned}
|m| & =m_{1}+m_{2}+\cdots+m_{n} ; \\
m+a & =\left(m_{1}+a, m_{2}+a, \ldots, m_{n}+a\right) ; \\
r^{m} & =\prod_{j=1}^{n} r_{j}^{m_{j}} ; \\
(m+a)^{b} & =\prod_{j=1}^{n}\left(m_{j}+a\right)^{b} ; \quad \text { and } \\
m & \leqq l \text { means } m_{j} \leqq l_{j}, j=1,2, \ldots, n .
\end{aligned}
$$

We also make use of the following notation: if $f$ is a function of $r=\left(r_{1}, \ldots, r_{n}\right)$, then

$$
f^{(m)}(r)=\frac{\partial^{|m|}}{\partial r_{1}^{m_{1}} \cdots \partial r_{n}^{m_{n}}} f(r)
$$

and

$$
\int f(r) d r=\int f(r) d r_{1} d r_{2} \cdots d r_{n}
$$

THEOREM 5.1. Suppose that $f=\sum_{m} a_{m} z^{m}$ is analytic in the polydisc

$$
D=\left\{z \in C^{n}:\left|z_{j}\right|<1 ; j=1, \ldots, n\right\},
$$

that $\left\{\sum_{|m|=k}\left|a_{m}\right|^{2}\right\}^{1 / 2} \leqq A_{k}$ for $z$ in $D(k=0,1, \ldots)$, and that for each multi-index $m$

(i) $f^{(m)}$ has a continuous extension to $D^{-}$;

(ii) $f^{(m)}(1, \ldots, 1)=0$. 
Then a necessary and sufficient condition to conclude that $f=0$ is that

$$
\sum_{k=1}^{\infty} k^{-3 / 2} \log A_{k}
$$

diverge.

The following two lemmas contain some of the computations used repeatedly in the proof of Theorem 5.1.

LEMMA 5.2. If $\sum_{|m| \geqq k} \sum_{l \leqq m} x(m, l)$ is a convergent series of nonnegative terms, then

$$
\sum_{|l| \leqq k} \sum_{m \geqq l} x(m, l) \leqq \sum_{|m| \geqq k} \sum_{l \leqq m} x(m, l)
$$

Proof.

$$
\begin{aligned}
\sum_{|l|=k} \sum_{m \geqq l} x(m, l) & =\sum_{m} \sum_{|l|=k, l \leqq m} x(m, l) \\
& =\sum_{|m| \geqq k} \sum_{|l|=k, l \leqq m} x(m, l) \leqq \sum_{|m| \geqq k} \sum_{l \leqq m} x(m, l) .
\end{aligned}
$$

LemMA 5.3. If $\sum_{|m|=j}\left|a_{m}\right|^{2} \leqq A_{j}^{-2}(j=1,2, \ldots)$ and (4) diverges, then for $k=1,2$, ... and $b \geqq 1 / n$

$$
\sum_{j=k}^{\infty} \sum_{|m|=j}\left|a_{m}\right|^{2}(m+1)^{b} \leqq c k^{n b+2} A_{k}^{-2}
$$

for some constant $c$.

Proof. If $|m|=j \geqq 1,(m+1)^{1 / n} \leqq(j+n) / n$ by the inequality of the arithmetic and geometric means. Hence $(m+1)^{b} \leqq(j / n+1)^{n b} \leqq(2 j)^{n b}$ and

$$
\sum_{|m|=j}\left|a_{m}\right|^{2}(m+1)^{b} \leqq(2 j)^{n b} \sum_{|m|=j}\left|a_{m}\right|^{2} \leqq(2 j)^{n b} A_{j}^{-2}
$$

Thus it suffices to prove that

$$
\sum_{j=k}^{\infty} j^{n b} A_{j}^{-2} \leqq c k^{n b+2} A_{k}^{-2} \quad(k=1,2, \ldots) .
$$

Since (4) diverges, it follows that $\lim _{k \rightarrow \infty} s_{k}=\infty$ (as in the proof of Theorem 3.4). Hence there exists an integer $k_{0} \geqq 1$ such that for $k \geqq k_{0}, s_{k} \geqq \frac{1}{2} n b+1$. So for $j>k \geqq k_{0}$,

$$
\frac{\log A_{j}-\log A_{k}}{\log j-\log k} \geqq s_{k} \geqq \frac{1}{2} n b+1 .
$$

And $A_{k} A_{j}^{-1} \leqq\left(k j^{-1}\right)^{(n b+2) / 2}$. So that

$$
\begin{aligned}
\sum_{j=k}^{\infty} j^{n b} A_{j}^{-2} & =A_{k}^{-2}\left(k^{n b}+\sum_{j=k+1}^{\infty} A_{k}^{2} A_{j}^{-2} j^{n b}\right) \\
& \leqq A_{k}^{-2} k^{n b+2} \sum_{j=1}^{\infty} j^{-2} .
\end{aligned}
$$


This proves the lemma for $k \geqq k_{0}$. By choosing $c$ large enough we see that the lemma is true for $k \geqq 1$.

Proof of Theorem 5.1. First suppose that (4) converges. Then by Theorem 3.4 there is a function $g(w) \not \equiv 0$ of one complex variable such that $g$ is in $F\left(A_{k}\right)$ and $g^{(k)}(1)=0$ for $k=0,1, \ldots$ Let $f(z)=g\left(z_{1}\right)$ for $z=\left(z_{1}, \ldots, z_{n}\right)$ in the polydisc $D$. Then $\left\{\sum_{|m|=k}\left|a_{m}\right|^{2}\right\}^{1 / 2} \leqq A_{k}$ for $z$ in $D(k=0,1, \ldots)$ and $f$ satisfies (i) and (ii) but $f \not \equiv$.

Now suppose that (4) diverges. We wish to show that $f \equiv 0$. Notice that since $f$ is analytic in each variable separately, it is enough to show that $f(r)=0$ for all $r=\left(r_{1}, \ldots, r_{n}\right)$ with $0<r_{j}<1, j=1, \ldots, n$. Fix such an $r$ and define a function of one complex variable by

$$
\phi(w)=\left(\left(1-r_{1}\right) w+r_{1}, \ldots,\left(1-r_{n}\right) w+r_{n}\right) .
$$

Then $\phi$ maps the open unit disc into $D$ (and the closed unit disc into $D^{-}$), $\phi(1)$ $=(1, \ldots, 1)$ and $\phi(0)=r$. Let

$$
g(w)=f(\phi(w))=\sum_{k=0}^{\infty} b_{k} w^{k}
$$

Then $g$ is analytic in the open unit disc,

$$
g^{(k)}(w)=\sum_{|m|=k} f^{(m)}(\phi(w)) r^{m}
$$

has a continuous extension to $D^{-}$and $g^{(k)}(1)=0$ for $k=0,1, \ldots$ We will show later that $\left|b_{k}\right| \leqq c k^{n+1} A_{k}^{-1}$ for $k=0,1, \ldots$ Assuming that this has been done, we let $\bar{A}_{k}=A_{k} k^{-(n+1)}, k=1,2, \ldots$ Then $\left\{b_{k} \bar{A}_{k}\right\}$ is bounded and $g$ is in $F\left(\bar{A}_{k}\right)$. Now $\log \bar{A}_{k}=\log A_{k}-(n+1) \log k$ is convex in $\log k$ and $\sum_{k=1} k^{-3 / 2} \log \bar{A}_{k}$ diverges since (4) diverges. It follows that $F\left(\bar{A}_{k}\right)$ is quasi-analytic and $g \equiv 0$. In particular $f(r)=f(\phi(0))=g(0)=0$.

Thus it suffices to show that $\left|b_{k}\right| \leqq c k^{n+1} A_{k}^{-1}$. To simplify notation, let

$$
x(l, m)=\prod_{j=1}^{n} C_{m_{j, l}, l_{j}}\left(1-r_{j}\right)^{l_{j}} r_{j}^{m_{j}-l_{j}} .
$$

Notice that $\sum_{l \leqq m} x(l, m)=1$. Furthermore

$$
\begin{aligned}
g(w) & =\sum_{m} a_{m} \sum_{l \leqq m} x(l, m) w^{|l|}=\sum_{l} \sum_{m \geqq l} a_{m} x(l, m) w^{|l|} \\
& =\sum_{k=0}^{\infty} \sum_{|l|=k} \sum_{m \geqq l} a_{m} x(l, m) w^{k} .
\end{aligned}
$$

Thus we conclude that

Finally

$$
b_{k}=\sum_{|l|=k} \sum_{m \geqq l} a_{m} x(l, m)
$$

$$
\left|b_{k}\right| \leqq\left\{\sum_{|l|=k} \sum_{m \geqq l} x(l, m)(m+1)^{-2}\right\}^{1 / 2}\left\{\sum_{|l|=k} \sum_{m \geqq l}\left|a_{m}\right|^{2} x(l, m)(m+1)^{2}\right\}^{1 / 2} .
$$


So by Lemma 5.2

$$
\begin{aligned}
\left|b_{k}\right| & \leqq\left\{\sum_{|m| \geqq k} \sum_{l \leqq m} x(l, m)(m+1)^{-2}\right\}^{1 / 2}\left\{\sum_{|m| \geqq k} \sum_{l \leqq m}\left|a_{m}\right|^{2} x(l, m)(m+1)^{2}\right\}^{1 / 2} \\
& =\left\{\sum_{|m| \geqq k}(m+1)^{-2}\right\}^{1 / 2}\left\{\sum_{|m| \geqq k}\left|a_{m}\right|^{2}(m+1)^{2}\right\}^{1 / 2} \\
& \leqq c\left\{\sum_{j=k}^{\infty} \sum_{|m|=j}\left|a_{m}\right|^{2}(m+1)^{2}\right\}^{1 / 2} .
\end{aligned}
$$

And by Lemma 5.3

$$
\left|b_{k}\right| \leqq c_{1}\left\{k^{2 n+2} A_{k}^{-2}\right\}^{1 / 2}=c_{1} k^{n+1} A_{k}^{-1} .
$$

We conclude this paper by observing that, as in the one variable case, if $f(z)$ $=\sum_{m} a_{m} z^{m}$ is analytic in $D, \sum_{|m|=k}\left|a_{m}\right|^{2} \leqq A_{k}^{-2}(k=1,2, \ldots)$ and (4) diverges, then $f^{(l)}$ already has a continuous extension to $D^{-}$for all $l$. To see this

$$
f^{(l)}(z)=\sum_{m \geqq l} \frac{m !}{(m-l) !} a_{m} z^{m-l}
$$

and

$$
\sum_{m \geqq l} \frac{m !}{(m-l) !}\left|a_{m}\right| \leqq \sum_{m}\left|a_{m}\right|(m+1)^{l}
$$

which is finite by computations similar to those above. Thus (5) converges uniformly on $D^{-}$.

\section{BIBLIOGRAPHY}

1. S. Bochner and A. E. Taylor, Some theorems on quasianalyticity for functions of several variables, Amer. J. Math. 61 (1939), 303-329.

2. L. Carleson, Sets of uniqueness for functions regular in the unit circle, Acta Math. 87 (1952), 325-345.

3. B. I. Korenbljum, Conditions of nontriviality of certain classes of functions analytic in a sector, and problems of quasianalyticity, Dokl. Akad. Nauk SSSR 166 (1966), 1046-1049 =Soviet Math. Dokl. 7 (1966), 233-236.

4. - Quasianalytic classes of functions in a circle, Dokl. Akad. Nauk SSSR 164 (1965), 36-39 = Soviet Math. Dokl. 6 (1965), 1155-1158.

5. S. Mandelbrojt, Séries adhérentes. Régularisation des suites. Applications, GauthierVillars, Paris, 1952.

6. - Séries de Fourier et classes quasi-analytiques de fonctions, Gauthier-Villars, Paris, 1935.

7. R. E. A. C. Paley and N. Wiener, Fourier transforms in the complex domain, Amer. Math. Soc. Colloq. Publ., Vol. 19, Amer. Math. Soc., Providence, R. I., 1934; reprinted 1967.

8. B. Rodrigues-Salinas, Funciones con momentos nulos, Rev. Acad. Ci. Madrid 49 (1955), 331-368.

UNIVERSITY OF MiCHIGAN,

ANN ARbor, MichigAn 